OPEN ACCESS

Edited by:

Laure S. Fournier,

Assistance Publique Hopitaux De

Paris, France

Reviewed by:

Amit Sethi,

Indian Institute of Technology

Bombay, India

Nandita Maria DeSouza, Institute of Cancer Research (ICR),

United Kingdom

*Correspondence:

Ghulam Nabi

g.nabi@dundee.ac.uk

Specialty section:

This article was submitted to

Cancer Imaging and

Image-directed Interventions,

a section of the journal

Frontiers in Oncology

Received: 13 July 2021 Accepted: 26 October 2021 Published: 23 November 2021

Citation:

Wei C, Zhang Y, Zhang X, Ageeli W,

Szewczyk-Bieda M, Serhan J,

Wilson J, Li C and Nabi G (2021)

Prostate Cancer Gleason Score From

Biopsy to Radical Surgery: Can

Ultrasound Shear Wave Elastography

and Multiparametric Magnetic

Resonance Imaging Narrow the Gap?

Front. Oncol. 11:740724.

doi: 10.3389/fonc.2021.740724

\section{Prostate Cancer Gleason Score From Biopsy to Radical Surgery: Can Ultrasound Shear Wave Elastography and Multiparametric Magnetic Resonance Imaging Narrow the Gap?}

\author{
Cheng Wei ${ }^{1}$, Yilong Zhang ${ }^{2}$, Xinyu Zhang ${ }^{3}$, Wael Ageeli ${ }^{1,4}$, Magdalena Szewczyk-Bieda ${ }^{5}$, \\ Jonathan Serhan ${ }^{5}$, Jennifer Wilson ${ }^{6}$, Chunhui $\mathrm{Li}^{2}$ and Ghulam Nabi ${ }^{{ }^{*}}$ \\ 1 Division of Imaging Sciences and Technology, School of Medicine, University of Dundee, Dundee, United Kingdom, \\ ${ }^{2}$ School of Science and Engineering, University of Dundee, Dundee, United Kingdom, ${ }^{3}$ Division of Population Health and \\ Genomics, University of Dundee, Dundee, United Kingdom, ${ }^{4}$ Diagnostic Radiology Department, College of Applied Medical \\ Sciences, Jazan University, Jazan, Saudi Arabia, ${ }^{5}$ Department of Clinical Radiology, Ninewells Hospital, Dundee, \\ United Kingdom, ${ }^{6}$ Department of Pathology, Ninewells Hospital, Dundee, United Kingdom
}

Objectives: To investigate the impact of ultrasound shear wave elastography (USWE) and multiparametric magnetic resonance imaging (mpMRI) in predicting a change in biopsyassigned Gleason Score (GS) after radical surgery for localised prostate cancer (PCa).

Method: A total of 212 men opting for laparoscopic radical prostatectomy (LRP) between September 2013 and June 2017 were recruited into this study. All the participants had 12core transrectal ultrasound (TRUS) biopsies and imaging using USWE and mpMRI before radical surgery. The predictive accuracy for imaging modalities was assessed in relation to upgrading and downgrading of PCa GS between the biopsies and radical prostatectomy using Student's t-test and multivariable logistic regression analyses. A decision analysis curve was constructed assessing the impact of nomogram on clinical situations using different thresholds of upgrading probabilities.

Results: Most GS 6 diseases on biopsies were upgraded on radical surgery (37/42, 88.1\%). Major downgrading was seen in GS 8 category of disease (14/35; 37.1\%), whereas no alteration was observed in GS 7 on biopsies in most men (55/75; 73.3\%). In univariate analysis, higher preoperative prostate-specific antigen (PSA) $(p=0.001)$, higher prostate-specific antigen density (PSAD) ( $p=0.002)$, stiffer USWE lesions $(p=0.009)$, and higher prostate imaging-reporting and data system (PIRADS) $(p=0.002)$ on mpMRI were significant predictors of upgrading. In multivariate logistic regression analyses, only PSA $(p=0.016)$ and USWE-measured tissue stiffness $(p=0.029)$ showed statistical significance in predicting upgrading.

Conclusions: Measurement of tissue stiffness using USWE in clinically localised PCa can predict upgrading of GS and has the potential to improve patient management options.

Keywords: prostate cancer, ultrasound shear wave elastography, multiparametric MRI, PIRADS, radical prostatectomy, prostate biopsy 


\section{HIGHLIGHTS}

$\bigcirc$ Ultrasound shear wave elastography can significantly predict upgrading of biopsy-assigned Gleason Score in prostate cancer following radical surgery.

$\bigcirc$ Ultrasound shear wave elastography is an emerging technology based on measurement of tissue stiffness.

$\bigcirc$ USWE-measured tissue stiffness can impact decision analysis based on different probabilities of Gleason Score upgrading from biopsies to radical surgery in prostate cancer.

\section{INTRODUCTION}

The histological Gleason Score (GS) obtained using 12-core transrectal ultrasound (TRUS) biopsy informs risk stratification and counselling of clinically localised prostate cancer (PCa) patients regarding various treatment options. The approach has significant limitations, as a large discrepancy exists between biopsy and postoperative radical prostatectomy GS in approximately $40 \%$ of all localised PCa patients especially for those with biopsy GS 6 disease (1-3). GS upgrading after radical surgery is also associated with poor disease prognostic factors such as extracapsular extension (ECE) and higher rates of biochemical recurrences (4). Thus, predicting GS prior to treatment of PCa becomes crucial, and the role of imaging as marker is less understood. The role of imaging in the detection and characterisation of PCa is now well-established (4). Prebiopsy multiparametric magnetic resonance imaging (mpMRI) has been widely applied to increase biopsy accuracy, particularly over the last decade (5-8). However, there is still a burgeoning interest in investigating the role that imaging can play in predicting underestimated GS in biopsies. This will help in accurately assessing prognosis, treatment selection, and decision-making.

Recently, ultrasound shear wave elastography (USWE) has emerged as a promising imaging modality in the detection and characterisation of localised PCa (9-11). USWE can assess tissue stiffness of the whole prostate including cancerous tissue. USWE measures the shear wave speed generated by specialised ultrasound transducers through the target organs. Under imaging, the speed of these scattered shear waves is shown as a colour-coded dynamic map of tissue stiffness (presented as Young's modulus) in real time $(12,13)$. The USWE-based imaging approach not only provides characterisation of clinically significant $\mathrm{PCa}$ (9) but also predicts biochemical recurrence on follow-up (14).

Although previous studies have focused on other multifactorial analyses and nomograms to predict GS change after radical surgery $(3,15-21)$, USWE or mpMRI, key imaging

\footnotetext{
Abbreviations: GS, Gleason Score; TRUS, transrectal ultrasound; PCa, prostate cancer; mpMRI, multiparametric magnetic resonance imaging; USWE, ultrasound shear wave elastography; $\mathrm{kPa}$, kilopascals; PSA, prostate-specific antigen; PSAD, prostate-specific antigen density; ESUR, European Society of Uro-radiology; PIRADS, prostate imaging-reporting and data system.
}

modalities have seldom been considered as potential imaging markers to predict GS upgrading or downgrading in PCa (22).

The aim of this study was to assess the impact of imaging markers [tissue stiffness using USWE and prostate imagingreporting and data system (PIRADS) using mpMRI] in predicting a change in biopsy-assigned GS after radical surgery for localised prostate cancer. Furthermore, we aimed to quantify the additional benefits that imaging information may bring to the already known and reported clinicopathological parameters through the construction of nomogram and decisionanalysis curves.

\section{MATERIALS AND METHODS}

\section{Study Cohort}

Two hundred and twelve patients opting for laparoscopic radical prostatectomy between September 2013 and June 2017 were recruited into this study. All patients were confirmed to have $\mathrm{PCa}$ on 12-core TRUS biopsies. In brief, transrectal ultrasound imaging of prostates was performed. After measuring size of prostate gland, local anaesthetic agent was infiltrated from the base to apex. Prostate was divided into 12 regions (as per our protocol) including lateral and paramedian regions. Each region was biopsied and sent for histopathology. Participants were then scanned using two imaging modalities: mpMRI and USWE preoperatively. The images from mpMRI were assessed, and abnormal areas were classified using PIRADS score by two uroradiologists. The USWE images were analysed, and a quantitative cancer stiffness estimation in kilopascals $(\mathrm{kPa})$ was made. Patients' age at the time of radical surgery, prostate-specific antigen (PSA), prostate weight, prostate-specific antigen density (PSAD), biopsy GS, number of positive cores, maximum of cancer in cores, clinical stage, and postoperatively pathological GS were collected and analysed. Prostate specimens were sectioned in a $3 \mathrm{D}$ printed patient specific mould and analysed by two experienced study pathologists including the co-author (JW with more than 5 years' experience) $(23,24)$. Figure 1 shows the flow of participants recruited to the study. Table 1 presents baseline patient characteristics.

\section{USWE Protocol and Acquisition}

All USWE images were obtained using a transrectal endocavitory ultrasound transducer (SuperSonic Imagine, Aix en Provence, France) with patients being in either lithotomy or lateral position the day before the scheduled radical surgery. USWE mode was activated, and prostate gland elastograms were obtained from the cranial to caudal direction for each lobe of the prostate. All regions were scanned as described in our previously published protocol (11). Guidelines for clinical practice have been framed based on data emanating from many centres (25). Each patient's prostate gland was scanned transrectally; USWE images were acquired in transverse planes from the base to apex with a gap of 4-6 $\mathrm{mm}$. The most suspicious cancer located in the planes was marked and reconstructed offline into 3D images. Suspicious areas for cancer were scanned by rotating the transducer in 


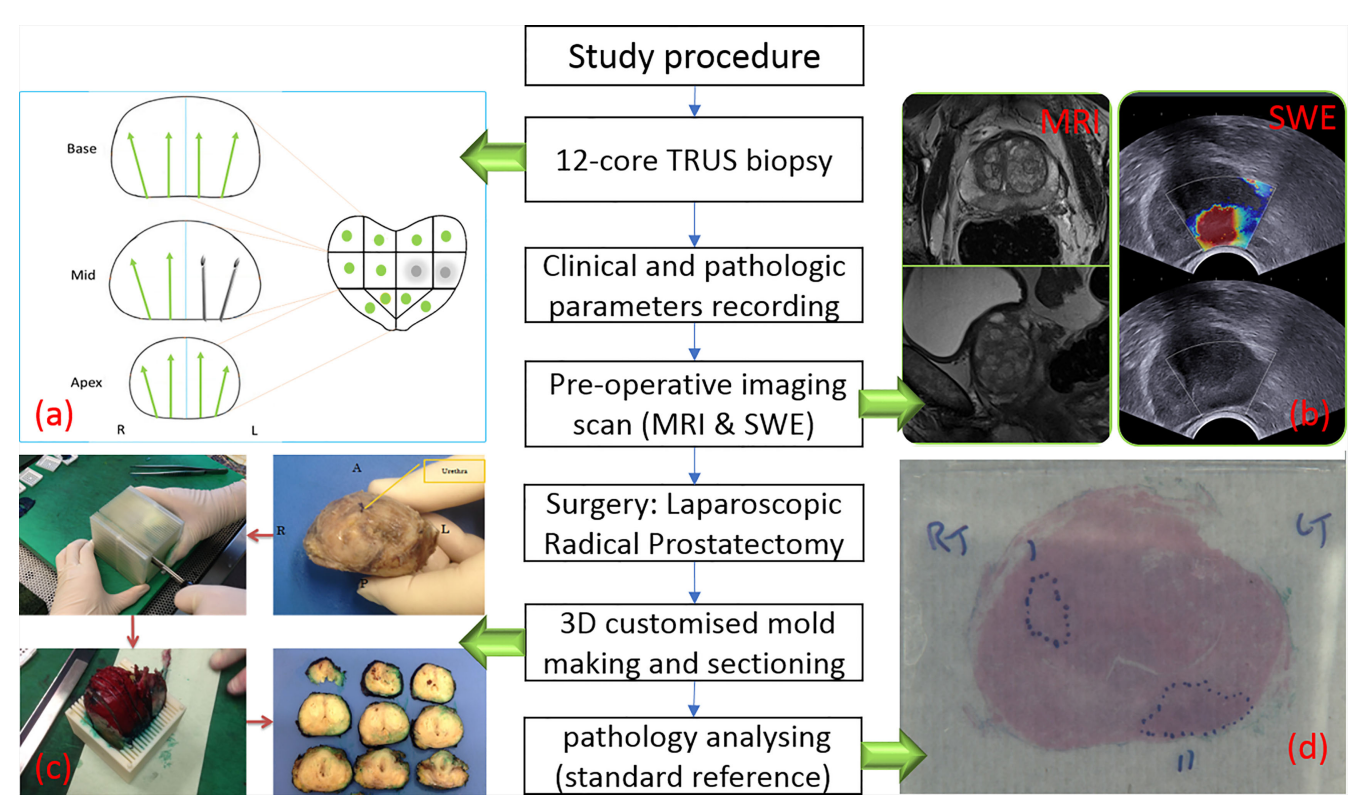

FIGURE 1 | Flow chart of study procedure. (A) TRUS biopsy result with two positive biopsy cores. (B) One suspicious lesion in peripheral zone is shown in MRI (left) and SWE (right) images. (C) Post-prostatectomy specimen sectioning in steps (23). (D) Histopathology photo after analysing.

different directions to confirm abnormalities and to perform measurements of their sizes. Three stiffness measurements of shear wave speed in $\mathrm{m} / \mathrm{s}$ or Young's modulus in $\mathrm{kPa}$ using pseudo-colour maps were obtained independently by three researchers. The ratio between abnormal and normal areas were also recorded (Figure 1B).

\section{MRI Protocol and PIRAD Score}

MRI scan of each patient was performed using $3 \mathrm{~T}$ scanners (TIM Trio, Siemens, Erlangen, Germany) 6-8 weeks after the prostate biopsy procedure (26). The MRI protocol was derived from the European Society of Uro-radiology (ESUR) guidelines 2012 (27) for PCa detection; PIRADS v2.0 was applied in this study, and only PIRAD $\geq 3$ lesions on MRI were marked and PIRADS 1 and 2 were taken as negative findings. All MR images were analysed and scored by two experienced uro-radiologists (MS-B and JS); both the radiologists were blinded to patients' clinicopathology data.

\section{Statistical Analyses}

A two-stage logistic regression process was used to investigate the explanatory factors that could predict upgrading of GS 6 or 7 $(3+4)$ and downgrading of GS $7(4+3)$ and above on biopsy. First, univariate logistic regression was applied to examine associations between single explanatory factor and the outcomes, respectively. Absolute percentages of each variable, univariate odds ratio (OR), 95\% CI of univariate OR, and p-value were presented. Multivariate logistic regression was then applied to assess and adjust for significant predictive factors regarding patient characteristics. The predictive factors in the multivariate logistic regression model were a combination of significantly associated factors from the bi-variate logistic regression.
Age, PSA, PSAD, maximum percentage of cancer in the core, and prostate gland weight, and quantitatively assessed stiffness using USWE were treated as continuous variables; clinical stage, number of positive cores for cancer, PIRADS scores, and GS were treated as ordinal variables. The reference groups of those predictive factors were set if they were considered as a meaningful reference of that variable. Adjusted OR, 95\% CI of adjusted OR, and p-value were derived after multivariate logistic regression.

In addition, logistic regression model coefficients were used to derive a nomogram predicating the probability of GS upgrading or downgrading from biopsy. Non-informative or nonsignificant variables in univariate logistic regression for GS upgrading were removed. The bias-corrected calibrated values were generated from internal validation based on 200 bootstrap resamples. A decision-analysis curve was constructed assessing the impact of the nomogram using different threshold probabilities of upgrading or downgrading of GS. All analyses were performed using SPSS 22 (IBM Corporation, New York, USA) and R software (v 3.5.3). The alpha level was set at 0.05 to determine two-tailed significance.

\section{RESULTS}

\section{Change in GS in the Cohort From Biopsy to Radical Surgery}

A detailed map of biopsy GS and radical prostatectomy specimen GS is shown in Table 2. A Sankey diagram in Figure $\mathbf{2}$ presents same data in an alternate way. No change in Gleason Score was seen in $47.2 \%$ of all the cases $(100 / 212)$. Out of the 42 cases with 
TABLE 1 | Patient characteristics

\begin{tabular}{|c|c|}
\hline \multicolumn{2}{|l|}{ Age (years) } \\
\hline Median (IQR) & $67.0(63.8-72.0)$ \\
\hline Mean (SD) & $67.2(5.7)$ \\
\hline Range & $44.0-77.0$ \\
\hline \multicolumn{2}{|c|}{ Prostate-specific antigen ( $\mathrm{ng} / \mathrm{ml})$} \\
\hline Median (IQR) & $9.4(7.1-12.5)$ \\
\hline Mean (SD) & $11.4(7.6)$ \\
\hline Range & $0.1-47.7$ \\
\hline \multicolumn{2}{|l|}{ Clinical stage (\%) } \\
\hline$\leq \mathrm{T} 2 \mathrm{a}$ & $148(69.8 \%)$ \\
\hline $\mathrm{T} 2 \mathrm{~b} / \mathrm{c}$ & $44(20.8 \%)$ \\
\hline T3 & $20(9.4 \%)$ \\
\hline \multicolumn{2}{|c|}{ Biopsy Gleason Score (\%) } \\
\hline$\leq 6$ & $42(19.8 \%)$ \\
\hline $7(3+4)$ & $75(35.4 \%)$ \\
\hline $7(4+3)$ & $39(18.4 \%)$ \\
\hline$>7$ & $56(26.4 \%)$ \\
\hline \multicolumn{2}{|c|}{ No. of positive cores } \\
\hline Median (IQR) & $4.0(2.0-7.0)$ \\
\hline Mean (SD) & $4.8(3.3)$ \\
\hline Range & $1.0-14.0$ \\
\hline \multicolumn{2}{|c|}{ Maximum percentage of cancer pre-core (\%) } \\
\hline Median (IQR) & $50.0(20.0-80.0)$ \\
\hline Mean (SD) & $50.4(30.0)$ \\
\hline Range & $5.0-100.0$ \\
\hline \multicolumn{2}{|c|}{ The interval from biopsy to SWE (days) } \\
\hline Median (IQR) & $102.5(83-118)$ \\
\hline Mean (SD) & $102.6(27.3)$ \\
\hline Range & $46-189$ \\
\hline \multicolumn{2}{|c|}{ The interval from biopsy to MRI (days) } \\
\hline Median (IQR) & $43(35-48)$ \\
\hline Mean (SD) & $43.8(11.2)$ \\
\hline Range & $21-78$ \\
\hline \multicolumn{2}{|c|}{ Radical prostatectomy weight (g) } \\
\hline Median (IQR) & $59.5(47.5-76.5)$ \\
\hline Mean (SD) & $66.9(29.4)$ \\
\hline Range & $31.0-207.0$ \\
\hline
\end{tabular}

GS 6 disease on biopsy, the majority $(37 / 42,88.1 \%)$ was upgraded following radical surgery. GS $3+4$ disease on biopsies remained stable in most of the cases $(55 / 75 ; 73.3 \%)$ and so did the GS $9(4+5$ or $5+4)$ disease in most cases $(76.2 \%)$. GS $4+3$ disease on biopsies had downgrading in one-third of cases $(13 / 39,33.3 \%)$. Most downgrading $(13 / 35 ; 37.1 \%)$ was seen in biopsy GS $8(3+5,4+4,5+3)$ category disease.

\section{Multifactorial Analysis of GS Change at Radical Surgery}

Table 3 shows the preoperative clinical and imaging parameters in men with and without upgraded GS at radical surgery. As seen, the data indicate that upgraded patients had a higher PSA level $(\mathrm{p}=0.001)$ and a greater PSAD $(\mathrm{p}=0.002)$, stiffer cancerous tissue as estimated by USWE ( $\mathrm{p}=0.009$ ), and higher PIRADS 4/5 score $(p=0.002)$. The results also showed a trend that upgraded patients were older $(\mathrm{p}=0.130)$, with more positive cores $(\mathrm{p}=$ 0.608), maximum percentage of cancer in a given core (0.071), and smaller prostates $(\mathrm{p}=0.806)$, but none of these variables were statistically significant. In multivariate logistic regression analyses (Table 4), higher stiffness values at USWE ( $p=0.029)$ and higher PSA level $(\mathrm{p}=0.016)$ predicted upgrading from biopsy GS $\leq 7(3+4)$ to $\mathrm{GS} \geq 7(4+3)$ after radical surgery.
The PIRADS score at mpMRI failed to maintain the same significance $(\mathrm{p}<0.05)$ in both univariate analysis $(\mathrm{p}=0.056)$ and multivariate analysis $(\mathrm{p}=0.068)$.

\section{Nomogram Construction, Validation, and Defining Thresholds for Decision Analysis}

Figures 3A1, A2 show a constructed nomogram predicting the upgrading of GS from biopsies to radical surgery with or without USWE data. Longer scales indicate a higher percentage of impact, and larger points suggest probability of upgrading. PSA level had the greatest impact in both nomograms. USWE counted as the second highest impact factor for GS upgrading. The nomograms were then internally validated using 200 bootstrap samples, and internal calibration curves were highlighted (Figures 3B1, B2). The calibration curves based on internal validation results are set for the probability of prediction at different levels. As seen, the curves demonstrated excellent agreement between the prediction according to the nomogram and actual observation. Decision analysis assumed that the threshold probability of a change in GS at which the clinician or patient would make an informed decision weighing the relative harms of a false-positive and a false-negative prediction using USWE information. A range of threshold probabilities was shown at which the magnitude of benefits of USWE was compared with no USWE information (Figure 4). The net benefit for the model using USWE was slightly higher but not quantitively proved at various thresholds compared with the model without USWE (blue $v s$. red line). The mean size of lesions from USWE was $16.1 \pm 7.2 \mathrm{~mm}$ (range from 7.4 to $44.8 \mathrm{~mm})$.

\section{DISCUSSION}

This was the first study to assess the role of both USWE and mpMRI in predicting change in biopsy-assigned GS following radical surgery in men presenting with clinically localised PCa. A review of the literature showed only limited reports of mpMRI parameters used in predicting GS upgrading. Lai et al. (28) found that mpMRI findings could predict upgrading GS $3+3$ disease on first biopsies in men on active surveillance. Abd-Alazeez et al. (29) concluded that a patient with higher PIRADS score on mpMRI predicted a high likelihood of high GS disease at radical surgery in men with low-risk PCa in biopsy. No 3D fabricated moulds were used to orient imaging to histopathology in any of the reported studies, a clear contrast to the present study. Similar observations were made by our group in the past (22). In contrast and interestingly, Klotz et al. (30) observed in a randomised multicentre prospective trial that adding MRI to clinicopathological factors did not boost the prediction ability of biopsy-assigned GS. In our study, we observed a high number of patients with PIRADS 4 and 5 in upgraded than not-upgraded patients [28.1\% (27/96) vs. 5.0\% (1/20), p = 0.002], but it was not a significant predictor in either univariate logistic regression model $(\mathrm{p}=0.056)$ or multivariate logistic model $(\mathrm{p}=0.068)$. There are no reports in the literature of USWE imaging being used in predicting change in GS. Previous studies only reported 
TABLE 2 | Radical prostatectomy grades stratified by biopsy Gleason Scores.

\begin{tabular}{|c|c|c|c|c|c|c|c|}
\hline \multirow[t]{2}{*}{ LRP GS } & & \multicolumn{5}{|c|}{ Biopsy GS } & \multirow[t]{2}{*}{ Total } \\
\hline & & $<7$ & $7(3+4)$ & $7(4+3)$ & 8 & $9-10$ & \\
\hline \multirow[t]{4}{*}{6} & Count & 5 & 0 & 0 & 0 & 0 & 5 \\
\hline & $\%$ within LRP GS & $100 \%$ & $0.0 \%$ & $0.0 \%$ & $0.0 \%$ & $0.0 \%$ & $100 \%$ \\
\hline & \% within Biopsy GS & $11.9 \%$ & $0.0 \%$ & $0.0 \%$ & $0.0 \%$ & $0.0 \%$ & $2.4 \%$ \\
\hline & $\%$ of total & $2.4 \%$ & $0.0 \%$ & $0.0 \%$ & $0.0 \%$ & $0.0 \%$ & $2.4 \%$ \\
\hline \multirow[t]{4}{*}{$7(3+4)$} & Count & 29 & 55 & 13 & 5 & 0 & 102 \\
\hline & $\%$ within LRP GS & $28.4 \%$ & $53.9 \%$ & $12.7 \%$ & $4.9 \%$ & $0.0 \%$ & $100 \%$ \\
\hline & \% within biopsy GS & $69.0 \%$ & $73.3 \%$ & $33.3 \%$ & $14.3 \%$ & $0.0 \%$ & $48.1 \%$ \\
\hline & $\%$ of total & $13.7 \%$ & $25.9 \%$ & $6.1 \%$ & $2.4 \%$ & $0.0 \%$ & $48.1 \%$ \\
\hline \multirow[t]{4}{*}{$7(4+3)$} & Count & 3 & 5 & 16 & 9 & 2 & 35 \\
\hline & $\%$ within LRP GS & $8.6 \%$ & $14.3 \%$ & $45.7 \%$ & $25.7 \%$ & $5.7 \%$ & $100 \%$ \\
\hline & \% within biopsy GS & $7.1 \%$ & $6.7 \%$ & $41.0 \%$ & $25.7 \%$ & $5.7 \%$ & $16.5 \%$ \\
\hline & $\%$ of total & $1.4 \%$ & $2.4 \%$ & $7.5 \%$ & $4.2 \%$ & $0.9 \%$ & $16.5 \%$ \\
\hline \multirow[t]{4}{*}{8} & Count & 1 & 6 & 4 & 8 & 3 & 22 \\
\hline & $\%$ within LRP GS & $4.5 \%$ & $27.3 \%$ & $18.2 \%$ & $36.4 \%$ & $13.6 \%$ & $100 \%$ \\
\hline & \% within biopsy GS & $2.4 \%$ & $8.0 \%$ & $10.3 \%$ & $22.9 \%$ & $14.3 \%$ & $10.4 \%$ \\
\hline & $\%$ of total & $0.5 \%$ & $2.8 \%$ & $1.9 \%$ & $3.8 \%$ & $1.4 \%$ & $10.4 \%$ \\
\hline \multirow[t]{4}{*}{$9-10$} & Count & 4 & 9 & 6 & 13 & 16 & 48 \\
\hline & $\%$ within LRP GS & $8.3 \%$ & $18.8 \%$ & $12.5 \%$ & $27.1 \%$ & $33.3 \%$ & $100 \%$ \\
\hline & \% within biopsy GS & $9.5 \%$ & $12.0 \%$ & $15.4 \%$ & $37.1 \%$ & $76.2 \%$ & $22.6 \%$ \\
\hline & $\%$ of total & $1.9 \%$ & $4.2 \%$ & $2.8 \%$ & $6.1 \%$ & $7.5 \%$ & $22.6 \%$ \\
\hline \multirow[t]{4}{*}{ Total } & Count & 42 & 75 & 39 & 35 & 21 & 212 \\
\hline & $\%$ within LRP GS & $19.8 \%$ & $35.4 \%$ & $18.4 \%$ & $16.5 \%$ & $9.9 \%$ & $100 \%$ \\
\hline & \% within biopsy GS & $100 \%$ & $100 \%$ & $100 \%$ & $100 \%$ & $100 \%$ & $100 \%$ \\
\hline & $\%$ of total & $19.8 \%$ & $35.4 \%$ & $18.4 \%$ & $16.5 \%$ & $9.9 \%$ & $100 \%$ \\
\hline
\end{tabular}

LRP GS: laparoscopic radical prostatectomy Gleason Score; Biopsy GS: biopsy Gleason Score.

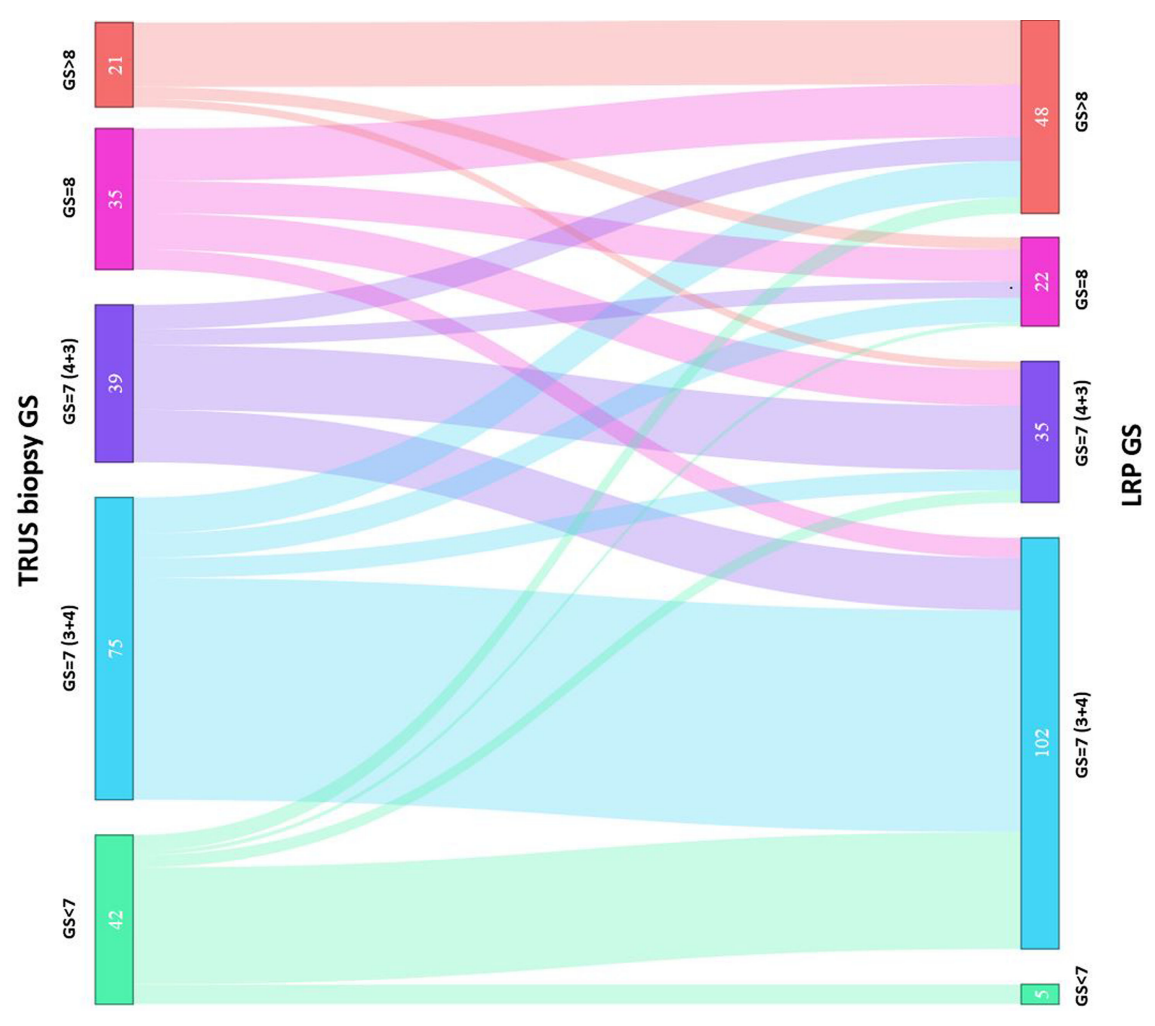

FIGURE 2 | Sankey diagram of comparison between biopsy Gleason Score and prostatectomy Gleason Score. 
TABLE 3 | Association of clinical and pathologic parameters with Gleason Score (GS) group: upgrading from biopsy GS $\leq 7(3+4)$ to GS $\geq 7(4+3)$ at radical prostatectomy.

\begin{tabular}{|c|c|c|c|c|}
\hline variables & Upgrade $(n=28)$ & No-upgrade $(n=89)$ & t value $(95 \% \mathrm{Cl})$ & p-value \\
\hline \multicolumn{5}{|l|}{ Age, year } \\
\hline Median (IQR) & $70.0(65.0-72.0)$ & $67.0(63.0-71.0)$ & $1.49(-0.61,4.14)$ & 0.130 \\
\hline Mean (SD) & $68.8(5.5)$ & $67.0(5.3)$ & & \\
\hline \multicolumn{5}{|l|}{ PSA, ng/ml } \\
\hline Median (IQR) & $11.5(7.3-16.2)$ & $8.8(6.9-10.5)$ & $3.34(1.98,7.78)$ & 0.001 \\
\hline Mean (SD) & $14.5(10.1)$ & $9.6(5.4)$ & & \\
\hline \multicolumn{5}{|c|}{ No. of positive cores } \\
\hline Median (IQR) & $3.0(1.0-5.0)$ & $3.0(2.0-5.0)$ & $0.47(-1.07,1.72)$ & 0.641 \\
\hline Mean (SD) & $4.0(3.3)$ & $3.6(2.8)$ & & \\
\hline \multicolumn{5}{|c|}{ Maximum \% cancer/core } \\
\hline Median (IQR) & $45.0(20.0-76.3)$ & $30.0(20.0-50.0)$ & $1.82(-0.98,23.02)$ & 0.071 \\
\hline Mean (SD) & $48.2(32.0)$ & $37.2(26.6)$ & & \\
\hline \multicolumn{5}{|c|}{ Pathology weight (continuous), gram } \\
\hline Median (IQR) & $62.0(51.0-76.5)$ & $63.0(47.6-86.8)$ & $1.24(-5.43,23.73)$ & 0.216 \\
\hline Mean (SD) & 64.7 (18.3) & $73.9(36.8)$ & & \\
\hline \multicolumn{5}{|c|}{ PSAD (continuous), $\mathrm{ng} / \mathrm{ml}^{2}$} \\
\hline Median (IQR) & $0.2(0.1-0.2)$ & $0.1(0.1-0.2)$ & $3.21(0.04,0.16)$ & 0.002 \\
\hline Mean (SD) & $0.3(0.2)$ & $0.2(0.10)$ & & \\
\hline \multicolumn{5}{|c|}{ Clinical stage (\%) } \\
\hline$\leq \mathrm{T} 2 \mathrm{a}$ & 16 & 69 & $1.83(-0.02,0.51)$ & 0.070 \\
\hline $\mathrm{T} 2 \mathrm{~b} / \mathrm{c}$ & 9 & 14 & & \\
\hline T3 & 3 & 6 & & \\
\hline \multicolumn{5}{|c|}{ USWE (continuous), kPa } \\
\hline Median (IQR) & 145.1 (128.8-168.5) & 128.7 (115.3-147.6) & $2.64(4.98,34.84)$ & 0.009 \\
\hline Mean (SD) & $154.2(42.3)$ & $134.3(31.4)$ & & \\
\hline \multicolumn{5}{|l|}{ PI-RADS } \\
\hline$\leq 3$ & 1 & 19 & $2.23(0.02,0.34)$ & 0.028 \\
\hline 4 and 5 & 27 & 69 & & \\
\hline Not reported & 0 & 1 & & \\
\hline
\end{tabular}

USWE as a promising diagnostic modality in the detection of clinically significant PCa $(9-11,31,32)$.

Preoperative PSA levels or PSAD are the most frequently analysed factors as predictor of GS change in the reported literature and were included in this study as well. From the reported publications $(19,21,28,33)$, it appears that PSA or PSAD performed consistently well, although in other studies, the significance was not as strong in comparison to other predictors $(34,35)$, but all the studies had used preoperative PSA in multivariate logistic regression models. In this study, PSA level was found to be one of the two significant parameters in multivariate logistic regression analysis.

Smaller prostate size was not a statistically significant predicting factor for GS upgrading in this study $(\mathrm{p}=0.086)$. This is similar to observations by other studies $(16,18-20)$, although Freedland et al. (36) showed that decreased prostate size was associated with higher Gleason grade, more aggressive behaviour, and higher biochemical recurrence rates.

In studies by Epstein et al. (19) and Gondo et al. (33), age, PSA level, prostate weight, and maximum cancer core

TABLE 4 | Univariate and multivariate logistic regression models to predict upgrading from biopsy GS $\leq 7(3+4)$ to GS $\geq 7(4+3)$ at radical prostatectomy.

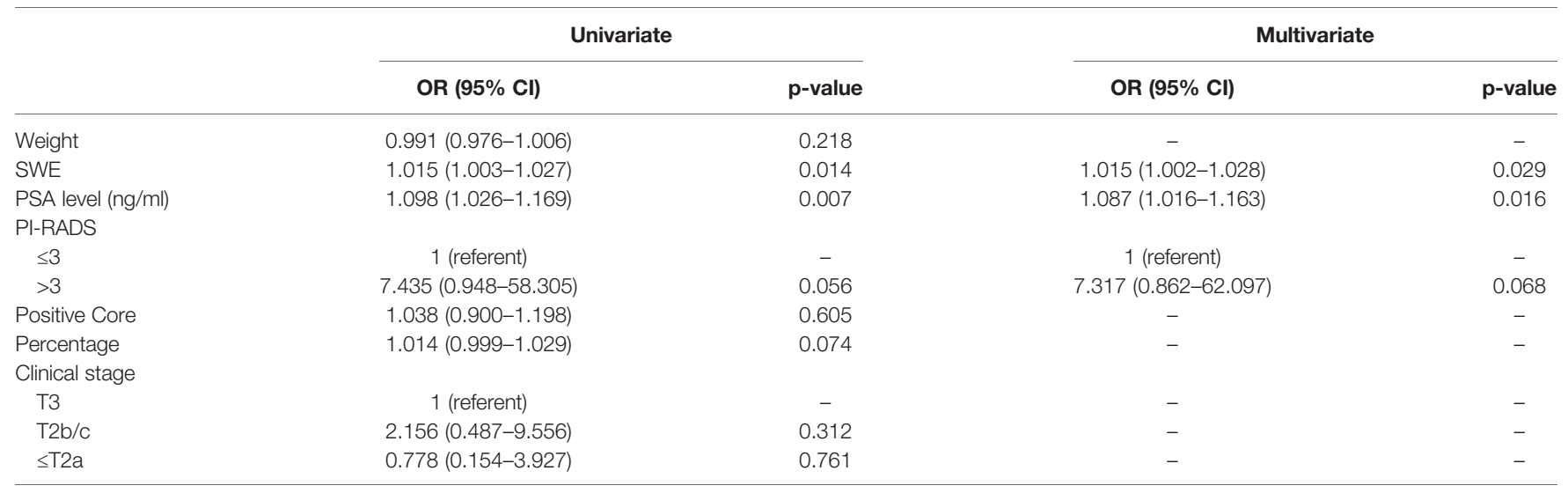

OR: odd ratio; Univariate and Multivariate analysis are two statistical analyses. Univariate involves the analysis of a single variable while multivariate analysis examines two or more variables. Most multivariate analysis involves a dependent variable and multiple independent variables. 

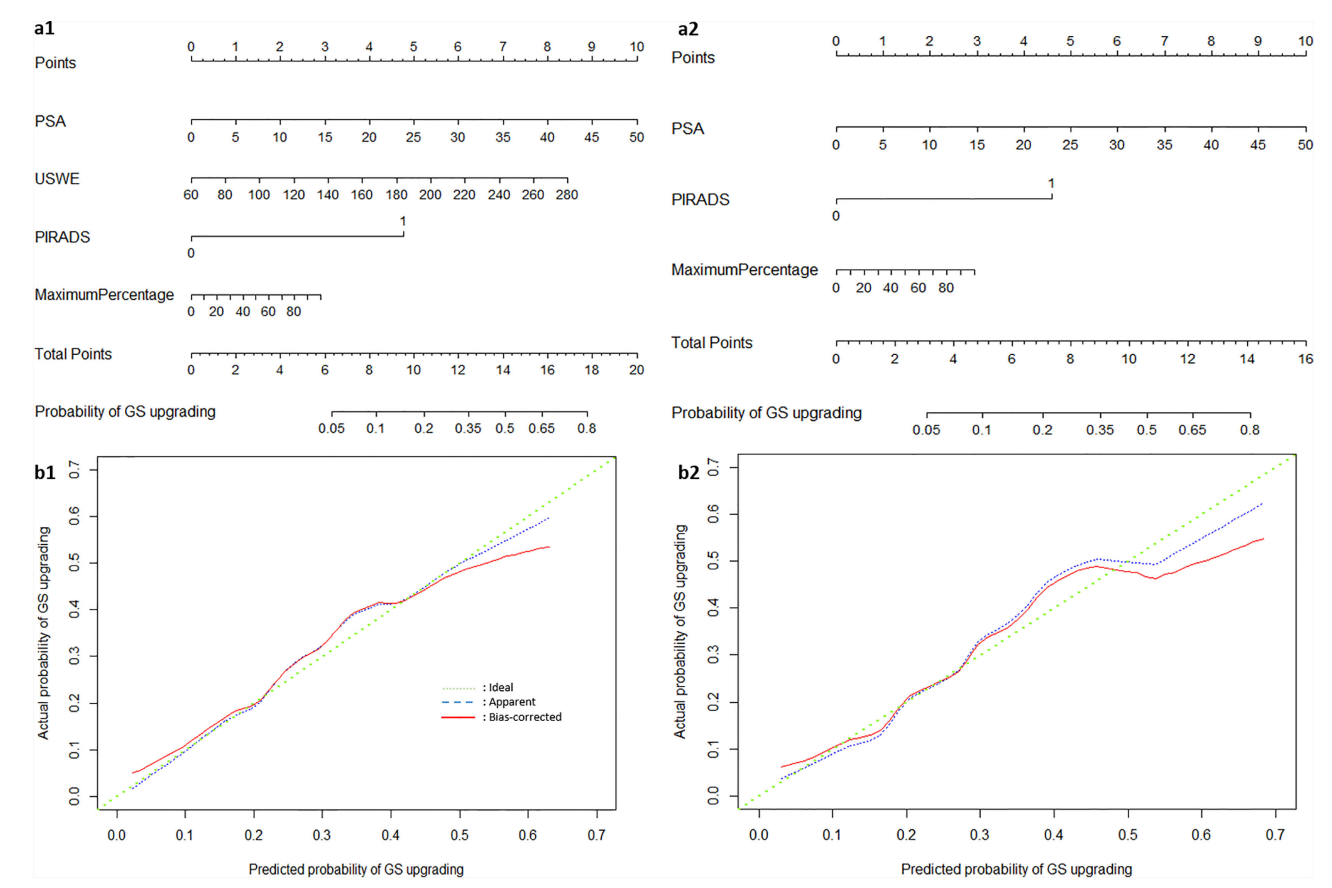

FIGURE 3 | The nomograms of Gleason Score upgrading prediction with (A1) and without USWE score (A2). Calibration plots of observed and predicted probability of GS upgrading with (B1) and without USWE score (B2).

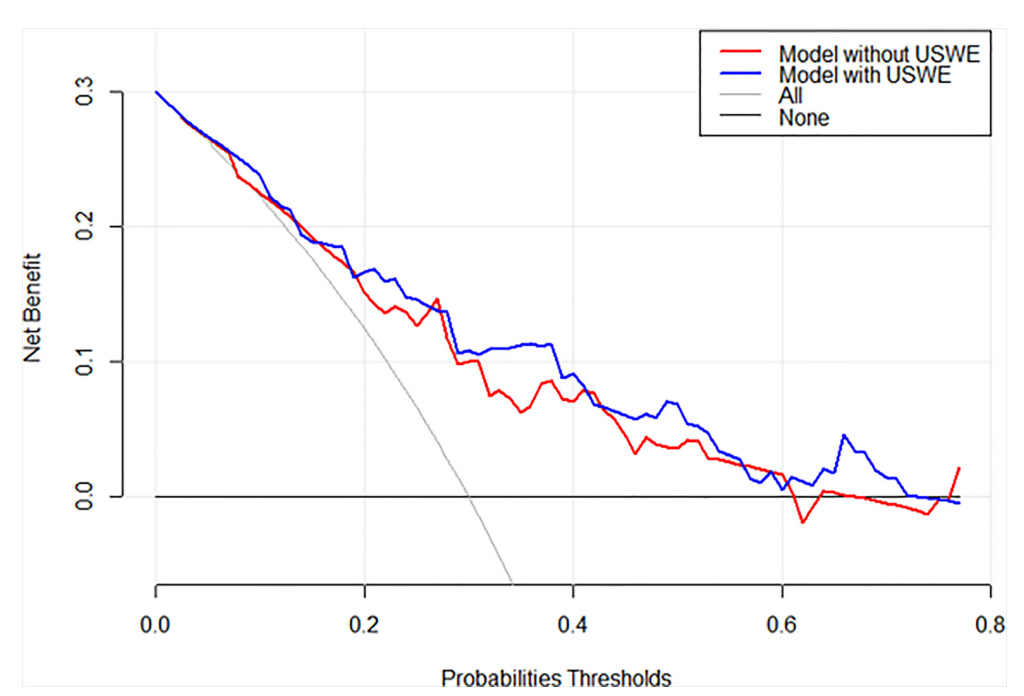

FIGURE 4 | Decision analysis demonstrated a high net benefit of USWE score model across a wide range of threshold probabilities. Prediction model without USWE score (red line); prediction model with USWE score (blue line).

involvement were all statistically significant predictors of downgrading. In our study, downgraded patients were more likely to have a lower PSA level $(8.1$ vs. $10.6 \mathrm{ng} / \mathrm{ml})$, but this was not statistically significant $(\mathrm{p}=0.075)$. Three studies summarised downgrading from biopsy GS $3+4$ to biopsy GS $<7$ at varying rates of $7.3 \%, 9.0 \%$, and $12.0 \%(19,33,37)$, respectively. No patients' postoperative GS was downgraded to GS $3+3$ in our study (see first raw of Table 2).

There were limitations worth mentioning in this study. First, this study recruited men with histologically confirmed PCa and only those opting for radical surgery. The focus of the study was to obtain a robust reference standard of histology from radical 
prostatectomy obtained specimens. We used both USWE and MRI imaging modalities in a preoperative setting. This was considered as the standard of care approach at the time of study; however, this had the potential of introducing a selection bias in the study. Second, the biopsy technique used in this study was 12-cores TRUS biopsy without targeting, and this has potentially created sampling error in the patients recruited to this study. Third, MRI scans were obtained after biopsies confirmed $\mathrm{PCa}$, and this might have introduced a detection bias in estimating PIRADS score. Finally, this was a single institutional study, and the findings require external validation and the reproducibility of the USWE technique (38). The use of USWE is not the standard of care, although guidelines and evidence are emerging in this area (39). The study was single centred with only operator performed USWE. Further reproducibility in multi-operator setting needs to be tested. We did not calculate the learning curve for this technology. Future studies could focus on the role of both USWE and/or MRItargeted biopsy in patients suspected of $\mathrm{PCa}$ and in predicting change in GS from biopsies to radical surgery.

Measurement of tissue stiffness using USWE in clinically localised PCa can predict upgrading of GS and better guide patient management options. This information may help in counselling patients opting for PCa therapy for localised disease.

\section{REFERENCES}

1. Kvåle R, Møller B, Wahlqvist R, Fosså SD, Berner A, Busch C, et al. Concordance Between Gleason Scores of Needle Biopsies and Radical Prostatectomy Specimens: A Population-Based Study. BJU Int (2009) 103 (12):1647-54. doi: 10.1111/j.1464-410X.2008.08255.x

2. Fernandes ET, Sund Aram CP, Long R, Soltani M, Ercole CJ. Biopsy Gleason Score: How Does it Correlate With the Final Pathological Diagnosis in Prostate Cancer? Br J Urol (1997) 79(4):615-7. doi: 10.1046/j.1464410X.1997.00126.x

3. Calio BP, Sidana A, Sugano D, Gaur S, Maruf M, Jain AL, et al. Risk of Upgrading From Prostate Biopsy to Radical Prostatectomy Pathology-Does Saturation Biopsy of Index Lesion During Multiparametric Magnetic Resonance Imaging-Transrectal Ultrasound Fusion Biopsy Help? J Urol (2018) 199(4):976-82. doi: 10.1016/j.juro.2017.10.048

4. Pinthus JH, Witkos M, Fleshner NE, Sweet J, Evans A, Jewett MA, et al. Prostate Cancers Scored as Gleason 6 on Prostate Biopsy are Frequently Gleason 7 Tumors at Radical Prostatectomy: Implication on Outcome. J Urol (2006) 176(3):979-84. doi: 10.1016/j.juro.2006.04.102

5. Anastasiadis AG, Lichy MP, Nagele U, Kuczyk MA, Merseburger AS, Hennenlotter J, et al. MRI-Guided Biopsy of the Prostate Increases Diagnostic Performance in Men With Elevated or Increasing PSA Levels After Previous Negative TRUS Biopsies. Eur Urol (2006) 50(4):738-49. doi: 10.1016/j.eururo.2006.03.007

6. Ehdaie B, Shariat SF. Magnetic Resonance Imaging-Targeted Prostate Biopsy: Back to the Future. Eur Urol (2013) 63(1):141-2; discussion 143-4. doi: 10.1016/j.eururo.2012.06.049

7. Moore CM, Robertson NL, Arsanious N, Middleton T, Villers A, Klotz L, et al. Image-Guided Prostate Biopsy Using Magnetic Resonance Imaging-Derived Targets: A Systematic Review. Eur Urol (2013) 63(1):125-40. doi: 10.1016/ j.eururo.2012.06.004

8. Kasivisvanathan V, Rannikko AS, Borghi M, Panebianco V, Mynderse LA, Vaarala MH, et al. MRI-Targeted or Standard Biopsy for Prostate-Cancer Diagnosis. N Engl J Med (2018) 378(19):1767-77. doi: 10.1056/ NEJMoa1801993

\section{DATA AVAILABILITY STATEMENT}

The datasets generated for this study are available on request to the corresponding author.

\section{ETHICS STATEMENT}

The studies involving human participants were reviewed and approved by East of Scotland Research Ethics Service (EoSRES) REC 1. The patients/participants provided their written informed consent to participate in this study.

\section{AUTHOR CONTRIBUTIONS}

GN and CW contributed to the conception and design of the study. $\mathrm{CW}$ and $\mathrm{YZ}$ organised the database. $\mathrm{YZ}$ and $\mathrm{XZ}$ performed the statistical analysis. CW wrote the first draft of the manuscript. CW, WA, MS-B, JS, JW, and CL corrected and rewrote sections of the manuscript. All authors contributed to the article and approved the submitted version.

9. Wei C, Li C, Szewczyk-Bieda M, Upreti D, Lang S, Huang Z, et al. Performance Characteristics of Transrectal Shear Wave Elastography Imaging in the Evaluation of Clinically Localized Prostate Cancer: A Prospective Study. J Urol (2018) 200(3):549-58. doi: 10.1016/j.juro.2018.03.116

10. Sigrist RMS, Liau J, Kaffas AE, Chammas MC, Willmann JK. Ultrasound Elastography: Review of Techniques and Clinical Applications. Theranostics (2017) 7(5):1303-29. doi: 10.7150/thno.18650

11. Ahmad S, Cao R, Varghese T, Bidaut L, Nabi G. Transrectal Quantitative Shear Wave Elastography in the Detection and Characterisation of Prostate Cancer. Surg Endosc (2013) 27(9):3280-7. doi: 10.1007/s00464-013-2906-7

12. Bercoff J, Tanter M, Fink M. Supersonic Shear Imaging: A New Technique for Soft Tissue Elasticity Mapping. IEEE Trans Ultrasonics Ferroelectrics Frequency Control (2004) 51(4):396-409. doi: 10.1109/TUFFC.2004.1295425

13. Bercoff J, Chaffai S, Tanter M, Sandrin L, Catheline S, Fink M, et al. In Vivo Breast Tumor Detection Using Transient Elastography. Ultrasound Med Biol (2003) 29(10):1387-96. doi: 10.1016/S0301-5629(03)00978-5

14. Wei C, Zhang Y, Malik H, Zhang X, Alqahtani S, Upreti D, et al. Prediction of Postprostatectomy Biochemical Recurrence Using Quantitative Ultrasound Shear Wave Elastography Imaging. Front Oncol (2019) 9(572). doi: 10.3389/ fonc. 2019.00572

15. Imamoto $\mathrm{T}$, Suzuki $\mathrm{H}$, Utsumi $\mathrm{T}$, Takano $\mathrm{M}$, Suyama $\mathrm{T}$, Kawamura $\mathrm{K}$, et al. External Validation of a Nomogram Predicting the Probability of Prostate Cancer Gleason Sum Upgrading Between Biopsy and Radical Prostatectomy Pathology Among Japanese Patients. Urology (2010) 76(2):404-10. doi: 10.1016/j.urology.2009.05.084

16. Davies JD, Aghazadeh MA, Phillips S, Salem S, Chang SS, Clark PE, et al. Prostate Size as a Predictor of Gleason Score Upgrading in Patients With Low Risk Prostate Cancer. J Urol (2011) 186(6):2221-7. doi: 10.1016/j.juro.2011.07.104

17. Corcoran NM, Hong MKH, Casey RG, Hurtado-Coll A, Peters J, Harewood L, et al. Upgrade in Gleason Score Between Prostate Biopsies and Pathology Following Radical Prostatectomy Significantly Impacts Upon the Risk of Biochemical Recurrence. BJU Int (2011) 108(8b):E202-10. doi: 10.1111/ j.1464-410X.2011.10119.x

18. Tilki D, Schlenker B, John M, Buchner A, Stanislaus P, Gratzke C, et al Clinical and Pathologic Predictors of Gleason Sum Upgrading in Patients 
After Radical Prostatectomy: Results From a Single Institution Series. Urologic Oncol: Semin Original Investigations (2011) 29(5):508-14. doi: 10.1016/ j.urolonc.2009.07.003

19. Epstein JI, Feng Z, Trock BJ, Pierorazio PM. Upgrading and Downgrading of Prostate Cancer From Biopsy to Radical Prostatectomy: Incidence and Predictive Factors Using the Modified Gleason Grading System and Factoring in Tertiary Grades. Eur Urol (2012) 61(5):1019-24. doi: 10.1016/j.eururo.2012.01.050

20. Gershman B, Dahl DM, Olumi AF, Young RH, McDougal WS, Wu C-L, et al. Smaller Prostate Gland Size and Older Age Predict Gleason Score Upgrading. Urologic Oncol: Semin Original Investigations (2013) 31(7):1033-7. doi: 10.1016/j.urolonc.2011.11.032

21. Xu N, Wu Y-P, Li X-D, Lin M-Y, Zheng Q-S, Chen S-H, et al. Risk of Upgrading From Prostate Biopsy to Radical Prostatectomy Pathology: Is Magnetic Resonance Imaging-Guided Biopsy More Accurate? J Cancer (2018) 9(19):3634-9. doi: 10.7150/jca.26791

22. Alqahtani S, Wei C, Zhang Y, Szewczyk-Bieda M, Wilson J, Huang Z, et al. Prediction of Prostate Cancer Gleason Score Upgrading From Biopsy to Radical Prostatectomy Using Pre-Biopsy Multiparametric MRI PIRADS Scoring System. Sci Rep (2020) 10(1):7722. doi: 10.1038/s41598-020-64693-y

23. Sheikh N, Wei C, Szewczyk-Bieda M, Campbell A, Memon S, Lang S, et al. Combined T2 and Diffusion-Weighted MR Imaging With Template Prostate Biopsies in Men Suspected With Prostate Cancer But Negative Transrectal Ultrasound-Guided Biopsies. World J Urol (2017) 35(2):213-20. doi: 10.1007/ s00345-016-1855-x

24. Wei C, Lang S, Bidaut L, Doull R, Huang Z, Nabi G, et al. Computer Aided Image Analysis and Rapid Prototyping Molds Using Patient-Specific MRI Data for Reliable Comparison Between Imaging and Histopathology of Radical Prostatectomy Specimens. Br J Surg (2014) 101:67-7.

25. Barr RG, Cosgrove D, Brock M, Cantisani V, Correas JM, Postema AW, et al. WFUMB Guidelines and Recommendations on the Clinical Use of Ultrasound Elastography: Part 5. Prostate. Ultrasound Med Biol (2017) 43(1):27-48.

26. Choi MH, Jung SE, Park YH, Lee JY, Choi Y-J. Multiparametric MRI of Prostate Cancer After Biopsy: Little Impact of Hemorrhage on Tumor Staging. Investig Magn Reson Imaging (2017) 21(3):139-47. doi: 10.13104/imri.2017.21.3.139

27. Barentsz J, Richenberg J, Clements R, Choyke P, Verma S, Villeirs G, et al. ESUR Prostate MR Guidelines 2012. Eur Radiol (2012) 22(4):746-57. doi: 10.1007/s00330-011-2377-y

28. Lai WS, Gordetsky JB, Thomas JV, Nix JW, Rais-Bahrami S. Factors Predicting Prostate Cancer Upgrading on Magnetic Resonance ImagingTargeted Biopsy in an Active Surveillance Population. Cancer (2017) 123 (11):1941-8. doi: $10.1002 /$ cncr.30548

29. Abd-Alazeez M, Ahmed HU, Arya M, Allen C, Dikaios N, Freeman A, et al. Can Multiparametric Magnetic Resonance Imaging Predict Upgrading of Transrectal Ultrasound Biopsy Results at More Definitive Histology? Urologic Oncol: Semin Original Investigations (2014) 32(6):741-7.

30. Klotz L, Loblaw A, Sugar L, Moussa M, Berman DM, Van der Kwast T, et al. Active Surveillance Magnetic Resonance Imaging Study (ASIST): Results of a Randomized Multicenter Prospective Trial. Eur Urol (2019) 75(2):300-9. doi: 10.1016/j.eururo.2018.06.025

31. Woo S, Kim SY, Cho JY, Kim SH. Shear Wave Elastography for Detection of Prostate Cancer: A Preliminary Study. Korean J Radiol (2014) 15(3):346-55. doi: $10.3348 / \mathrm{kjr} .2014 .15 .3 .346$
32. Boehm K, Salomon G, Beyer B, Schiffmann J, Simonis K, Graefen M, et al. Shear Wave Elastography for Localization of Prostate Cancer Lesions and Assessment of Elasticity Thresholds: Implications for Targeted Biopsies and Active Surveillance Protocols. J Urol (2015) 193(3):794-800. doi: 10.1016/ j.juro.2014.09.100

33. Gondo T, Poon BY, Matsumoto K, Bernstein M, Sjoberg DD, Eastham JA. Clinical Role of Pathological Downgrading After Radical Prostatectomy in Patients With Biopsy Confirmed Gleason Score $3+4$ Prostate Cancer. BJU Int (2015) 115(1):81-6. doi: 10.1111/bju.12769

34. Magheli A, Hinz S, Hege C, Stephan C, Jung K, Miller K, et al. Prostate Specific Antigen Density to Predict Prostate Cancer Upgrading in a Contemporary Radical Prostatectomy Series: A Single Center Experience. J Urol (2010) 183 (1):126-32. doi: 10.1016/j.juro.2009.08.139

35. Visapää H, Hotakainen K, Lundin J, Ala-Opas M, Stenman UH. The Proportion of Free PSA and Upgrading of Biopsy Gleason Score After Radical Prostatectomy. Urologia Internationalis (2010) 84(4):378-81. doi: $10.1159 / 000296302$

36. Freedland SJ, Isaacs WB, Platz EA, Terris MK, Aronson WJ, Amling CL, et al. Prostate Size and Risk of High-Grade, Advanced Prostate Cancer and Biochemical Progression After Radical Prostatectomy: A Search Database Study. J Clin Oncol (2005) 23(30):7546-54. doi: 10.1200/JCO.2005.05.525

37. Moussa AS, Kattan MW, Berglund R, Yu C, Fareed AK, Jones JS. A Nomogram for Predicting Upgrading in Patients With Low- and Intermediate-Grade Prostate Cancer in the Era of Extended Prostate Sampling. BJU Int (2010) 105(3):352-8. doi: 10.1111/j.1464-410X.2009. 08778.x

38. Harvey H, Morgan V, Fromageau J, O'Shea T, Bamber J, deSouza NM. Ultrasound Shear Wave Elastography of the Normal Prostate: Interobserver Reproducibility and Comparison With Functional Magnetic Resonance Tissue Characteristics. Ultrason Imaging (2018) 40(3):158-70. doi: 10.1177/ 0161734618754487

39. Barr RG. Just Because There is a Button Doesn't Mean it is Appropriate to Use or That it Will Give Accurate Information. J Ultrasound Med (2019) 0(0):15070. doi: 10.1002/jum.14990

Conflict of Interest: The authors declare that the research was conducted in the absence of any commercial or financial relationships that could be construed as a potential conflict of interest.

Publisher's Note: All claims expressed in this article are solely those of the authors and do not necessarily represent those of their affiliated organizations, or those of the publisher, the editors and the reviewers. Any product that may be evaluated in this article, or claim that may be made by its manufacturer, is not guaranteed or endorsed by the publisher.

Copyright (๑ 2021 Wei, Zhang, Zhang, Ageeli, Szewczyk-Bieda, Serhan, Wilson, Li and Nabi. This is an open-access article distributed under the terms of the Creative Commons Attribution License (CC BY). The use, distribution or reproduction in other forums is permitted, provided the original author(s) and the copyright owner(s) are credited and that the original publication in this journal is cited, in accordance with accepted academic practice. No use, distribution or reproduction is permitted which does not comply with these terms. 